Ann. Biol. anim. Bioch. Biophys., I968, 8 (2), 30I-3rr.

\title{
ÉTUDE DE L'ACTIVITÉ ÉLECTRIQUE DE LA MUSCULATURE LISSE DE L'ESTOMAC (RÉSEAU) CHEZ LES PETITS RUMINANTS. ACTION DE LA STIMULATION VAGALE
}

\author{
J.-P. ROUSSEAU (1) \\ Département de Neurophysiologie végétative, I. N.P.-C. N.R.S., \\ 31, Chemin Joseph-Aiguier, 13 -Marseille
}

(Directeur : M. Dussardier)

\section{SOMMATRE}

Nous avons enregistré in vivo chez 32 boucs et moutons l'activité électrique des fibres muscu. laires lisses superficielles du réseau (premier compartiment de l'appareil gastrique des Ruminants). Nous avons utilisé, soit des électrodes externes posées sur la séreuse, soit des micropipettes de verre extracellulaires remplies de $\mathrm{KCl} 3 \mathrm{M}$. De l'ensemble des résultats obtenus, trois faits doivent retenir l'attention :

- la fibre musculaire lisse du réseau présente une rythmicité permanente et intrinsèque, indépendante dans nos conditions expérimentales de toute commande nerveuse centrale;

- la stimulation périphérique du nerf vague ne déclenche pas, mais accélère cette activité propre de la musculature lisse de l'organe ;

- la latence de la réponse est de l'ordre de la demi-seconde. Les effets de la stimulation cessent rapidement après la fin de celle-ci.

La polarité positive et la forme des potentiels recueillis par microélectrodes externes sont discutées. Le problème des rapports fonctionnels existant entre la fibre musculaire lisse et les systèmes nerveux intrinsèque et extrinsèque dont elle dépend est posé.

\section{INTRODUCTION}

Le réseau constitue le premier compartiment de l'appareil gastrique des Ruminants. Sa motricité est caractérisée par des contractions biphasiques puissantes qui apparaissent environ toutes les minutes. Ces contractions rythmiques sont sous la dépendance étroite du système nerveux central : elles se produisent lorsque des influx

(1) Adresse actuelle : Laboratoire de Physiologie de la Reproduction. C. R. V. Z., 37 - Nouzilly. 
nerveux moteurs d'origine bulbaire parviennent aux estomacs par la voie des nerfs vagues (Dussardier, I96o ; BEGHELIII et coll., I96I ; LEEK, I963 ; IGGo et LEEK, I967). Aussi a-t-on qualifié ces contractions "d'extrinsèques ", par opposition aux petites contractions localisées ou "intrinsèques " qui apparaissent entre les précédentes et qui, contrairement à celles-ci, persistent après la bivagotomie (DUNCAN, I953; DuSSARDIER, I960). La motricité du réseau a surtout été étudiée à l'aide des techniques myographiques classiques, in vivo sur l'animal anesthésié ou éveillé et in vitro sur l'organe isolé.

Pour notre part, nous avons pensé que l'électromyographie pouvait apporter des renseignements plus précis sur le mode de dépendance de la musculature lisse du réseau vis-à-vis de son innervation extrinsèque. Nous avons enregistré in vivo l'activité électrique des fibres musculaires superficielles de cet organe et étudié les modifications provoquées par la stimulation vagale.

\section{TECHNIQUES EXPÉRIMENTALES}

\section{Préparation des animaux}

Les animaux ( 27 boucs et 5 moutons), à jeun depuis 36 heures, sont anesthésiés au Thiogénal (5-méthyl-thioéthyl 5-I méthyl butyl thiobarbiturate de sodium) injecté dans la veine jugulaire. L'anesthésie est entretenue par la perfusion de Nembutal dissous dans du chlorure de sodium à 9 p. I ooo ou par l'inhalation d'un mélange gazeux : oxygène-protoxyde d'azote-fluothane. Les deux vagues sont disséqués en région cervicale ; l'un d'eux est chargé sur une paire d'électrodes de stimulation. Après laparotomie dans le flanc gauche, l'ouverture du rumen permet de placer un ballon dans la lumière du réseau. La plaie du rumen et celle de l'abdomen sont refermées. Le réseau, très antérieur, est alors abordé par la voie transthoracique. Il est découvert après ablation du tiers inférieur des $6^{\mathrm{e}}$ et $7^{\mathrm{e}}$ côtes gauches et incision du diaphragme (BoST, 1958). Les bords musculaires de l'incision diaphragmatique sont suturés aux lèvres de la plaie cutanée. Le vide pleural est rétabli ; l'animal respire spontanément. Le réseau ainsi mis à nu est recouvert d'huile de paraffine à $3^{\circ} \mathrm{C}$ afin d'éviter sa dessication et son refroidissement. Enfin, nous curarisons l'animal par une injection intramusculaire de flaxédil (40 mg) et nous le plaçons sous respiration contrôlée chaque fois que les mouvements respiratoires entraînent une mobilisation excessive de l'estomac.

\section{Enregistrement}

La grosse difficulté est de conserver l'enregistrement de l'activité électrique malgré les déplacements du réseau, sollicité à la fois par ses propres contractions et par la respiration. Dans une première série d'expériences, nous avons utilisé des électrodes externes bipolaires posées sur la séreuse de l'organe. Chaque électrode est composée de deux fils de cuivre émaillés, de o,I $\mathrm{mm}$ de diamètre, dont les extrémités présentent entre elles un écartement variable, inférieur à deux millimètres. Cette tresse, spiralée sur plusieurs centimètres de longueur, reste suffisamment souple pour suivre les mouvements de la préparation, tout en exerçant une pression convenable sur le tissu. Dans une seconde série d'expériences, nous avons recueilli l'activité des fibres lisses au moyen de micropipettes extracellulaires, remplies de $\mathrm{KCl}_{3} \mathrm{M}$. Ces électrodes, montées flottantes selon la technique de WoODBURY et BRADY (1956), avaient à leur extrémité un diamètre de plusieurs microns. Dans les deux cas, les activités électriques ont été transmises à un amplificateur ayant une constante de temps de I seconde, puis visualisées sur l'écran d'un oscilloscope et enregistrées simultanément sur bande magnétique. Les variations de la pression intraréticulaire, transmises par veine liquide, sont transformées en différences de potentiel grâce à un capteur de type capacitif.

\section{Stimulation}

Nous utilisons des impulsions rectangulaires de I ms de durée et de Io $\mathrm{V}$ d'intensité, groupées en une salve d'une seconde. $\mathrm{La}$ fréquence des impulsions a été choisie selon les stimulations entre 8 et $4^{\circ}$ par seconde. 


\section{RÉSULTATS}

\section{Activité électrique spontanée}

Recueillie à 1'aide des électrodes externes bipolaires, cette activité se traduit par l'apparition de bouffées de potentiels durant de 80 à $200 \mathrm{~ms}$ et se répétant régulièrement à la cadence d'une à deux par seconde. L'amplitude des potentiels qui les composent varie de I00 à $500 \mu \mathrm{V}$ (fig. I A-B). Le plus souvent, lorsqu'elles sont recueillies en deux régions du réseau distantes de Io à $15 \mathrm{~mm}$, les bouffées de potentiels n'apparaissent pas en phase sous chaque électrode bipolaire. Sur des enregistrements de longue durée, on constate néanmoins qu'elles deviennent parfois synchrones pendant deux à trois secondes (fig. I A).

Enfin, une activité électrique beaucoup moins complexe a pu être enregistrée en rapprochant le plus possible l'une de l'autre les deux extrémités de l'électrode en contact avec l'estomac; elle est caractérisée par des potentiels triphasiques de $20 \mathrm{~ms}$ de durée, dont la fréquence de pulsation reste stable autour de 2 par seconde (fig. I C).
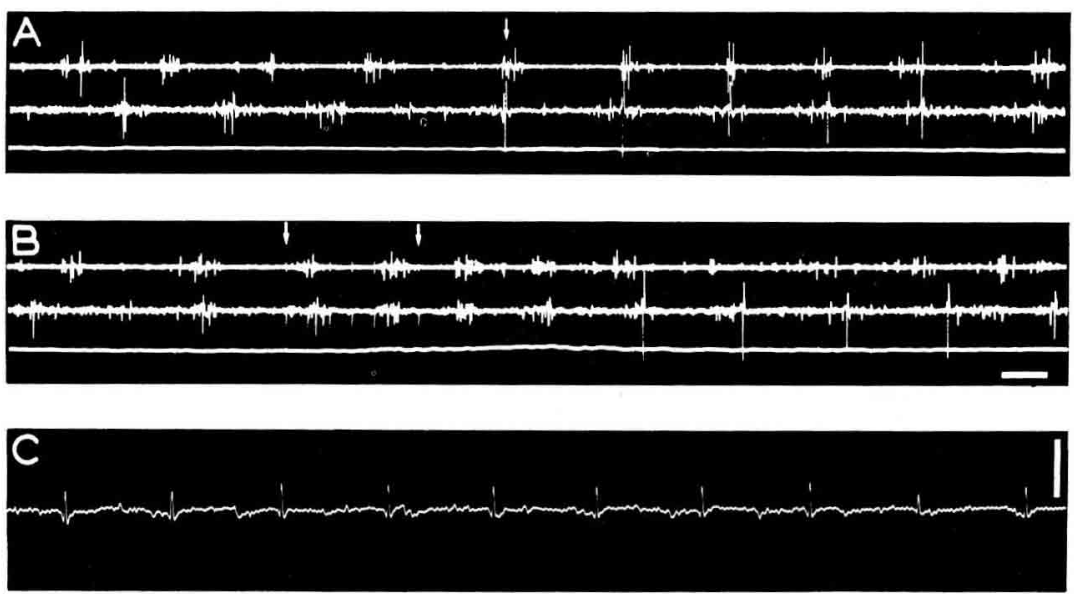

Fig. I. - Activité électrique enregistrée au niveau des fibres musculaires superficielles du réseau, a laide d'électrodes externes bipolaires

A et $B$ : Chacun des tracés montre le décours des activités électriques enregistrées au niveau de deux régions du réseau distantes de $15 \mathrm{~mm}$. En $\mathrm{A}$, les bouffées d'activité se présentent en phase sous chaque électrode bipolaire. Cette synchronisation, dont la flèche marque le début, persiste pendant plusieurs secondes. En B, la stimulation vagale (entre les deux flèches) provoque une légère contraction du réseau (ligne inférieure). Les bouffées d'activité apparaissent à une cadence plus rapide pendant et après la stimulation, mais leur mise en phase n'est pas la règle.

C : Activité rythmique vraisemblablement enregistrée au niveau d'une fibre musculaire.

Étalonnages : Amplitude : $0,5 \mathrm{mV}$ pour $\mathrm{A}$ et $\mathrm{B}, \mathrm{I} \mathrm{mV}$ pour $\mathrm{C}$ Temps : 0,4 s. pour A et $B, 0,2$ s. pour $C$.

Les potentiels recueillis par microélectrodes extracellulaires en réception monopolaire sont constitués par une phase initiale de dépolarisation lente durant de 400 à $500 \mathrm{~ms}$. Celle-ci donne naissance à une pointe positive de $\mathrm{I}$ à $\mathrm{I} 5 \mathrm{mV}$ selon le diamètre de la micropipette (fig. 2). Au cours d'un même enregistrement, ces potentiels de 
pointe n'ont pas toujours la forme simple que montre la figure $3 \mathrm{~A}$. Certains présentent un épaulement au début de leur phase de repolarisation (fig. 3 B-C), d'autres une encoche plus ou moins accentuée qui les divise alors en deux petites pointes (fig. 3 D-E-F). Les potentiels d'action recueillis sous la même électrode se répètent à un rythme régulier (fig. 2), mais leur fréquence varie selon les enregistrements de I à 2,5 par seconde. Cette périodicité pourrait suggérer que nous avons recueilli les modifications du champ électrique cardiaque. Il n'en est rien, car nous avons vérifié qu'il n'existait aucun rapport entre l'électrocardiogramme et l'activité obtenue au niveau de l'estomac, et la bivagotomie qui accélère le cœur n'est suivie d'aucune modification du rythme de la décharge (fig. 4 B).

L'hexaméthonium $(0,5 \mathrm{mg} / \mathrm{kg})$, injecté après la bivagotomie entraîne une légère diminution de la fréquence moyenne des potentiels recueillis (fig. $4 \mathrm{~B}$ ). Mais nous n'avons jamais pu conserver le même enregistrement jusqu'à la disparition de l'effet ganglioplégique, ni par conséquent vérifier le retour de l'activité à son niveau initial.
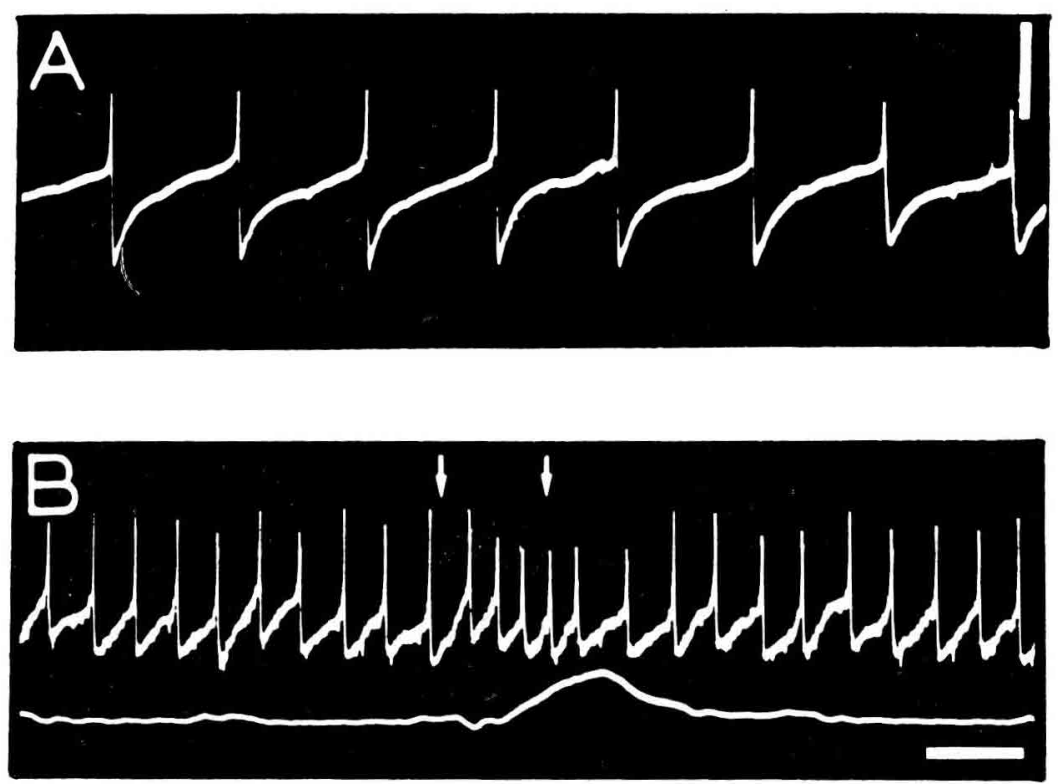

Fig. 2. - Enregistrement de l'activité électrique des fibres musculaires du réseau d l'aide de microélectrodes

A : Enregistrement de l'activité spontanée du muscle lisse.

Une déflexion vers le haut traduit une positivation de la pointe de la microélectrode.

B : Enregistrement de l'activité électrique du muscle lisse sur le tracé du haut et du mécanogramme sur le tracé du bas : une déflexion vers le haut traduit la contraction globale du réseau. La stimulation vagale (entre les flèches) entraîne une augmentation de la fréquence des potentiels recueillis.

Étalonnages : Amplitude : $4 \mathrm{mV}$

$0,4 s$ pour $\mathrm{A}$; I $s$. pour $\mathrm{B}$.

\section{Stimulation du nerf vague}

Seules des stimulations modérées de l'extrémité périphérique du vague ont été utilisées. Plus intenses, les stimulations provoquaient de fortes contractions réticu- 

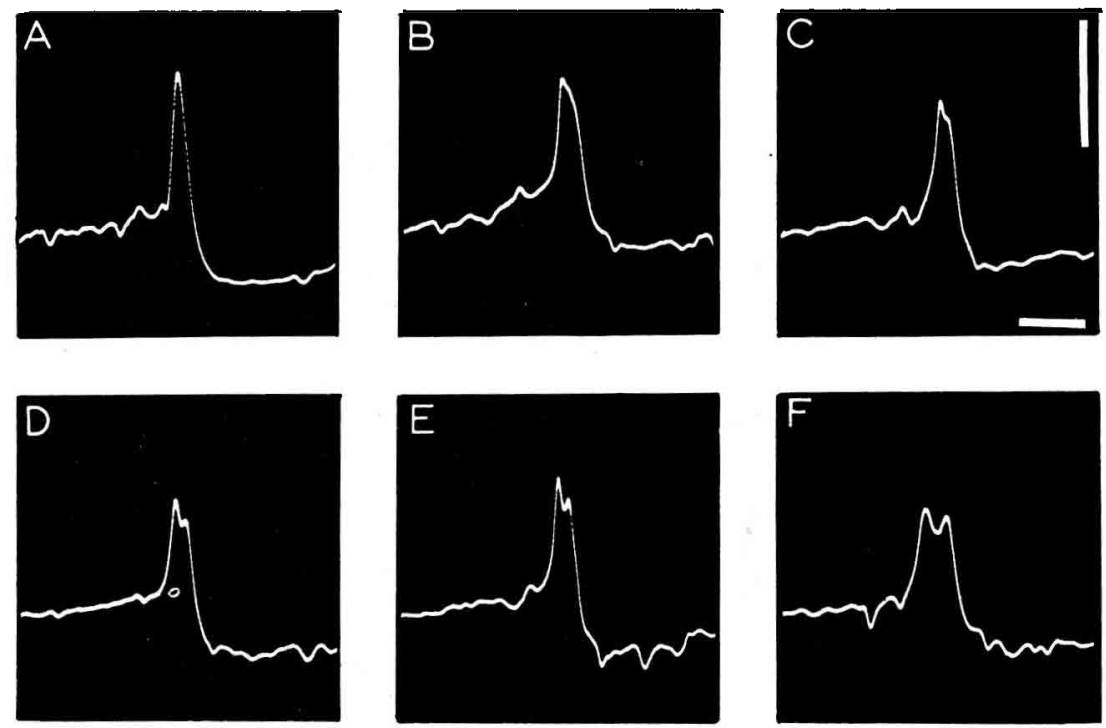

FIG. 3. - Différentes formes de potentiels de pointe

Ces potentiels ont été isolés à partir du même enregistrement de l'activité électrique spontanée du muscle lisse, recueillie par microélectrode externe.

A : forme simple la plus fréquemment rencontrée.

$\mathrm{B}$ et $\mathrm{C}$ : les potentiels présentent au cours de la phase de repolarisation un épaulement plus ou moins prononcé qui augmente la durée du potentiel de pointe.

$\mathrm{D}$ et $\mathrm{E}:$ une encoche divise le potentiel en deux petites pointes ; le phénomène est nettement marqué en $\mathrm{F}$. Étalonnages : Amplitude : $5 \mathrm{mV}$ Temps : $50 \mathrm{~m}$
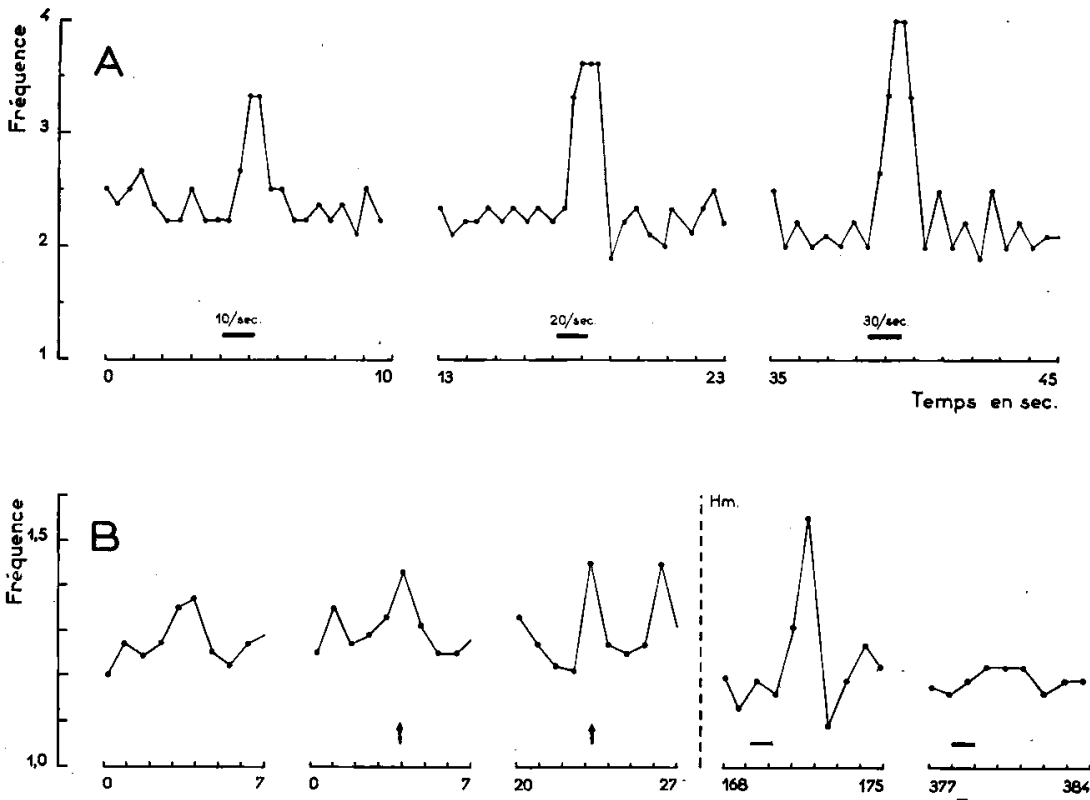

FIG. 4. - Evolution de la fréquence instantanée des potentiels recueillis par microélectrodes au niveau du réseau, au cours de deux enregistrements différents (A et B)

Nous avons porté en ordonnée l'inverse du temps qui sépare deux potentiels successifs.

A : Le rythme moyen de 2,2 potentiels par seconde est modifié par la stimulation vagale qui entraîne une augmentation de la fréquence des potentiels recueillis. Cette augmentation croît avec la fréquence des chocs électriques de stimulation.

B : Le rythme moyen de I,3 potentiel par seconde n'est pas modifié par la section successive des deux nerfs vagues (flèches). On injecte une solution d'hexaméthonium $\left(0,5 \mathrm{mg} / \mathrm{kg}\right.$ ) à la $66^{\circ}$ seconde $(\mathrm{Hm})$ par voie intraveineuse. La stimulation vagale à $t=I 69 \mathrm{~s}$ produit encore une faible augmentation de la fréquence des potentiels. Cet effet est totalement aboli à $t=378 \mathrm{~s}$. A noter la légère diminution de l'activité de base pendant l'action de l'hexaméthonium.

Ces diagrammes ont été établis à partir d'enregistrements identiques à ceux que montre la figure 2 B.

Annales de Biologie animale. - I968. 
laires qui déplaçaient les électrodes. Une stimulation juste supraliminaire entraîne une augmentation visible de l'activité recueillie par électrodes bipolaires externes : les bouffées de potentiels apparaissent à une cadence plus rapide pendant la phase active de la contraction gastrique (fig. I B). Mais il est plus facile d'apprécier quantitativement les résultats lorsqu'on enregistre l'activité à l'aide des microélectrodes extracellulaires. Dans ce cas, la fréquence des potentiels recueillis augmente progressivement jusqu'à un maximum qui s'avère d'autant plus élevé que la stimulation est plus forte (fig. $2 \mathrm{~B}$ ). Ainsi, pour une fréquence de stimulation de ro par seconde, l'accroissement maximum du rythme de la décharge atteint $50 \mathrm{p}$. Ioo de la valeur du rythme de base. Il dépasse 80 p. roo quand le nombre des chocs délivrés est de 30 par seconde (fig. $4 \mathrm{~A}$ ). Cette accélération semble résulter d'une augmentation de la vitesse de dépolarisation, qui affecte la variation lente du potentiel (fig. 2 B).

Il est difficile de définir avec précision la latence de la réponse, car la situation du premier choc entre deux potentiels successifs varie d'une stimulation à l'autre. On peut seulement affirmer qu'il s'écoule en moyenne une demi-seconde entre le début de la stimulation et l'apparition du premier potentiel dont la fréquence instantanée dépasse le niveau d'activité initial. Le dernier potentiel qui présente une telle caractéristique marque $1 \mathrm{a}$ fin de $1 \mathrm{a}$ réponse. Il apparaît 200 à $300 \mathrm{~ms}$ après la fin de la stimulation et précède d'autant le début du relâchement musculaire.

L'atropine $(2 \mathrm{cg})$ et 1'hexaméthonium $(0,5 \mathrm{mg} / \mathrm{kg})$ suppriment tout effet de la stimulation.

En conclusion, trois faits doivent retenir 1'attention :

$I^{0}$ la fibre musculaire lisse du réseau présente une rythmicité permanente et intrinsèque, indépendante dans nos conditions expérimentales de toute commande nerveuse centrale;

$2^{\circ}$ la stimulation vagale ne déclenche pas, mais accélère cette activité propre de la musculature lisse de l'organe;

$3^{\circ}$ la latence de la réponse est de l'ordre de la demi-seconde. Les effets de la stimulation cessent rapidement après la fin de celle-ci.

\section{DISCUSSION}

1. Il semble peu vraisemblable que nous ayons recueilli l'activité de cellules musculaires vasculaires bien que de nombreux vaisseaux irriguent la paroi gastrique. La musculature des artérioles est en effet sous la dépendance du système nerveux sympathique : la stimulation du nerf splanchnique ou 1'application locale d'adrénaline augmentent la fréquence des potentiels d'action au niveau des artérioles de la circulation mésentérique (SPEDEN, I964; STEEDMAN, I966) ou de la veine porte (FUNAKI et BoHR, I964); 1'acétylcholine par contre déprime l'activité des cellules musculaires vasculaires (STEEDMAN, I966). Or, nos résultats montrent que les structures que nous avons interrogées sont excitées par la stimulation vagale, et sont donc sous la dépendance du système nerveux parasympathique.

2. Polarité et forme des potentiels.

a) Les micropipettes extracellulaires que nous avons utilisées permettent de recueillir des potentiels d'action de polarité positive, alors qu'elles devraient en fait 
enregistrer une onde négative. Toutefois, les potentiels recueillis an niveau de l'intestin à l'aide d'électrodes externes, telles que les électrodes de pression ou de fines aiguilles d'acier, sont de même polarité que les potentiels intracellulaires (KOBAYAShI, Prosser et NAGaI, I967). On peut donc penser que, dans nos expériences, la micropipette extracellulaire se comporte comme une électrode de pression. Il est probable que sa pointe impose une déformation mécanique aux cellules musculaires sous-jacentes, les dépolarise et diminue la résistance de leur membrane. Puisqu'il existe des connexions de faible résistance entre les cellules (DEWEY, I965; KOIDE, I966), la micropipette peut être en contact électrique effectif avec l'intérieur des fibres musculaires adjacentes à celles que la pression a dépolarisées (BORTOFF, I967). Tout se passe comme si la pointe de l'électrode enregistrait à travers une résistance les variations de potentiel produites à l'intérieur de plusieurs fibres musculaires se comportant comme un syncitium.

b) Les potentiels d'action recueillis par microélectrodes extracellulaires ont une forme comparable à celle des potentiels obtenus sur certains autres muscles lisses : taenia coli (BuLBRING, I962), utérus sous imprégnation cestrogénique (MARSHALL, I959, I962). Une phase initiale de dépolarisation lente que l'on a l'habitude d'appeler " prépotentiel " ou " potentiel générateur " donne naissance à un potentiel de pointe. Cette lente dépolarisation entre les pointes est évidente. Sa durée détermine la fréquence de décharge qui est du même ordre pour le réseau que pour le taenia coli et l'utérus (I à 2 influx par seconde). Ces potentiels d'action n'apparaissent pas greffés sur les ondes lentes de type sinusoïdal, que des auteurs ont enregistrées à partir des fibres longitudinales de l'intestin (GoNEL,LA, I965) ou des cellules musculaires des artérioles mésentériques (STEEDMAN, I966). Nous ne pensons pas que 1'on puisse assimiler à ce type d'onde la phase initiale de dépolarisation lente que nous avons recueillie, car la cellule retourne à son potentiel de membrane initial immédiatement après chaque potentiel d'action : la pointe semble " effacer " la variation lente du potentiel (BURnstock, Holman et Prosser, I963). Le potentiel d'action recueilli fait plutôt penser à la décharge d'un récepteur de tension à adaptation nulle. Tout se passe comme si l'on avait un potentiel générateur stable; la phase initiale de dépolarisation lente aurait alors la valeur d'une reconstitution de ce potentiel générateur, mais ses rapports avec les potentiels d'action propagés sont difficiles à préciser. On peut également comparer ces variations de potentiel à celles enregistrées au niveau de certaines régions de l'utérus (extrémités cervicale et ovariennes), qui présentent une activité de type pace-maker (MARshaLI, I959).

Les modifications de forme qui affectent les potentiels de pointe ont déjà été observées par plusieurs auteurs sur des préparations de muscle isolé, au cours d'enregistrements de l'activité électrique spontanée (BUL,BRING, I957; HoLMAN, I958; TомiтA, I967). Elles peuvent également apparaître lorsqu'on applique un courant polarisant en une région du muscle voisine de l'électrode de réception (Tomit, I967). Il est possible qu'à l'endroit où deux faisceaux musculaires se rejoignent, la microélectrode ait recueilli simultanément les potentiels propagés le long de ces deux ensembles de fibres. L'épaulement ou l'encoche plus ou moins accentuée que montrent certains potentiels de pointe pourraient alors signifier qu'il existe un décalage de durée variable dans la réception successive des deux potentiels propagés. Il faudrait en conclure avec TomrTA ( $\left.196^{-}\right)$que la vitesse de conduction des potentiels le long 
d'un faisceau musculaire varie légèrement dans le temps et peut dépendre de l'état de polarisation de la membrane.

3. La mise en évidence d'une activité rythmique permanente s'oppose aux résultats obtenus sur l'animal éveillé par ITABISAshr et MaTsumoro (Ig66). Ces auteurs n'ont pas vu d'activité permanente, mais ont enregistré au moment de la contraction du réseau deux et parfois trois salves de potentiels qu'ils ont appelées grouping spike like deflections. Le niveau auquel nous avons fait porter notre analyse peut expliquer cette contradiction. Nous avons vraisemblablement recueilli l'électromyogramme de plusieurs fibres se comportant comme un syncitium, alors qu'ITABISASH et MATSUMOTO ont enregistré l'activité globale d'un nombre beaucoup plus important de fibres musculaires. Il est probable qu'une activité électrique existe entre les contractions du réseau, mais n'est pas apparente à cause des conditions de réception dans lesquelles ces auteurs se sont placés. I1 suffit en effet d'utiliser des électrodes plus fines pour enregistrer chez l'animal éveillé une activité permanente de faible amplitude (RUCKEBUSCH et LAPLACE, I967). Deux facteurs peuvent alors contribuer à révéler ou à accroître cette activité sous l'impulsion de la commande nerveuse centrale : l'augmentation de la fréquence de pulsation qui affecte chaque fibre musçulaire, associée à un degré plus élevé de synchronisation des activités élémentaires (Burnstock, Holman et Prosser, I963).

\section{Activité musculaire et système nerveux.}

a) Il est généralement admis que la plupart des muscles lisses sont stimulés par un étirement modéré (BOzLER, I947; BULBRING, I955). Cet étirement provoque une dépolarisation de la fibre et 1'apparition de potentiels d'action, ou une augmentation de leur fréquence lorsqu'ils existent déjà spontanément. Cette réaction est liée aux propriétés mêmes de la fibre musculaire et non à l'excitation des éléments nerveux intramuraux. Dans nos expériences, le réseau est légèrement distendu par un ballon. L'activité électrique que nous avons recueillie peut donc être la conséquence de la seule distension passive. Mais elle peut aussi traduire un état de tension musculaire entretenu par des influences tonigènes d'origine nerveuse. Elle permettrait alors d'analyser les rôles respectifs que pourraient jouer les systèmes nerveux extrinsèque et intrinsèque dans le maintien du tonus de l'organe.

Le problème revient en fait à déterminer si l'activité enregistrée est purement myogène ou neurogène. Dans ce dernier cas, l'activité des cellules ganglionnaires, aụ sein du plexus myentérique, peut être entretenue elle-même, soit par la voie vagale, soit par les neurones sensitifs intrapariétaux (PATON et VANE, I963; MORRISON et HABEL, I964). La possibilité d'activation des cellules du plexus par le nerf vague est montrée par les expériences de stimulation. Mais, la réalité d'une influence tonigène d'origine centrale n'est pas prouvée dans nos conditions expérimentales par les expériences de bivagotomie, laquelle n'est suivie d'aucune dépression de l'activité spontanée. Ellle existerait peut-être sans 1'action de l'anesthésie, car on a montré l'existence de quelques rares fibres motrices gastriques de type tonique empruntant la voie vagale chez l'animal éveillé (DUSSARDIER, I96o; RousSEAU, I966) ou légèrement anesthésié (IGGo et L/EEK, I967).

Quant aux ganglioplégiques, ils peuvent nous permettre d'apprécier l'action possible du système nerveux intrinsèque sur le tonus musculaire en bloquant la transmission entre cellules ganglionnaires. Cinq minutes après l'injection d'hexa- 
méthonium, on assiste effectivement à une légère diminution de la fréquence moyenne des potentiels recueillis. Cette dépression ne peut pas être le résultat d'un blocage de l'action vagale puisqu'on a pratiqué au préalable une bivagotomie. Elle semble donc bien signer une disparition de l'activité intrinsèque. On peut toutefois attribuer l'importance réduite de cette disparition au fait que dans nos conditions expérimentales, les cellules ganglionnaires sont déjà déprimées par les anesthésiques. Le fluothane, administré à des doses provoquant une anesthésie profonde, bloque le système nerveux périphérique autonome au niveau de l'estomac du Mouton (LEEK, I963). Les barbituriques sont aussi réputés pour déprimer partiellement l'activité des cellules ganglionnaires parasympathiques de la vessie (GARRET, I963). Il est donc difficile de mettre en évidence, dans nos conditions expérimentales, une action tonique du vague ou du plexus myentérique sur la musculature lisse du réseau.

b) Les modifications de 1'activité électrique observées à la suite des stimulations vagales soulèvent le problème des rapports morphologiques et fonctionnels existant entre la fibre musculaire lisse et la fibre nerveuse végétative. Il ressort de la bibliographie que le mode de dépendance des muscles lisses vis-à-vis de leur innervation extrinsèque varie avec les organes. La stimulation du nerf hypogastrique chez le Cobaye fait apparaître dans les fibres musculaires longitudinales du canal déférent des " potentiels de jonction " qui peuvent se sommer et produire un potentiel d'action (Burnstock et Holman, I96I). Au niveau de la musculature longitudinale du côlon, un choc unique appliqué sur le nerf pelvien peut donner naissance à des potentiels de pointe (GILILSPIE, Ig62). De même, la stimulation des fibres cholinergiques au sein de la musculature longitudinale de l'œsophage supérieur chez le Poulet produit une lente dépolarisation sur laquelle se greffe un potentiel d'action (OHAsHI et OHGA, I967). Mais nos résultats sont surtout à rapprocher de ceux obtenus par GoNELIA (I964) sur le duodénum du Lapin. Dans les deux cas, la stimulation du vague entraîne une augmentation de la fréquence des potentiels spontanés. Au niveau de l'intestin, la latence de la réponse, très variable, est toujours importante (elle dépasse souvent une seconde) et les effets produits par la stimulation persistent longtemps après la fin de celle-ci. Par contre, le réseau répond avec une latence plus courte, relativement constante, et l'effet obtenu cesse rapidement après la fin de la salve. Le rapport temporel qui existe entre l'activité nerveuse vagale et 1'activité musculaire apparaît donc plus étroit au niveau du réseau que du duodénum.

Dans l'état actuel de nos connaissances, il est difficile d'expliquer ces résultats : nous ignorons dans quelle mesure les ordres moteurs sont remaniés par les plexus nerveux intrapariétaux et de quelle manière les fibres postganglionnaires s'articulent avec les fibres musculaires du réseau. On sait seulement que les fibres préganglionnaires vagales se terminent sur un petit nombre de neurones myentériques situés en majeure partie au niveau de la " région centrale " des estomacs (MORRISoN et HABEx, Ig64). Celle-ci englobe le réseau, la gouttière osophagienne et la partie moyenne du sac dorsal du rumen, toutes structures qui présentent normalement de puissantes contractions commandées par le système nerveux central. Il serait intéressant dans un travail ultérieur d'enregistrer l'activité électrique des fibres lisses, au niveau du feuillet et de la caillette, car ces compartiments gastriques semblent moins soumis que le réseau à l'influence du système nerveux extrinsèque. 


\title{
SUMMARY
}

\author{
VAGAL STIMULATION AND ELECTRICAL ACTIVITY OF \\ THE STOMACH RETICULUM \\ IN THE SHEEP AND GOAT
}

The reticulum is the anterior pouch of the ruminant's forestomach. Its motility is characterized by biphasic cyclic contractions under vagal control and by located small contractions which not disappear after bivagotomy. We have used electromyographic techniques in order to investigate the relationships between the smooth muscle of the reticulum andi ts outer innervation. We have recorded the in vivo electrical activity of the muscular fibers of the external layer of the reticulum and studied the alterations induced by vagal stimulation.

The animals were anaesthetized with barbituric substances (Thiogenal, Nembutal) or fluothane. The reticulum was reached by opening the thorax and incising the diaphragm. Two types of electrodes were used ; bipolar external electrodes made of two insulated copper wires (tip diameter : o.I $\mathrm{mm}$ ) fixed on the serous; or extracellular, $\mathrm{KCl}$-filled glass microelectrodes flexibibly mounted. The cervical vagus was stimulated by a I $\mathrm{s}$ long volley of $\mathrm{I} \mathrm{ms}$ long stimuli. The intensity of the stimuli was to $\mathrm{V}$ and their frequency 8 to 4 per s.

External bipolar recordings showed bursts of stimuli of I or 2 per s. frequency and 80 to $200 \mathrm{~ms}$ duration (fig. I). In monopolar recordings, an initial slow wave of depolarization of 400 to $500 \mathrm{~ms}$ was followed by a positive spike of I to $15 \mathrm{mV}$ amplitude. The pulsating frequency was I to 2.5 per s. (fig. 2). Bivagotomy did not impair this rhythm, but vagal stimulation induced an increase in potential frequency (fig. 2 B). The response increased depending upon the frequency of the stimuli (fig. 4).

The following points should be stressed in our results :

- in our experimental conditions, the smooth fiber of the reticulum shows an autonomous rhythmic permanent activity, independant from any central nervous control ;

- vagal stimulation does not initiate, but emphasized this autonomous activity;

- the response latence is about half a second long. The effect of stimulation soon wears off when it is stopped.

The positive polarity and the form of potentials recorded by extracellular microelectrodes are discussed. The problem of vagal and intrinsic nervous influences on muscular tonus is raised. Functional relationship between the vagus and the smooth muscle of the reticulum are compared with other neuromuscular relationship previously studied.

\section{REMERCIEMEN'TS}

Nous remercions le Professeur M. DUSSARDIER pour ses conseils et ses commentaires, ainsi que l'I. N. R. A. pour son aide matérielle.

\section{RÉFÉRENCES BIBLIOGRAPHIQUES}

Beghelli V., Borgatti G., Parmeggiani P. L., i96I. Sulla funzione del nucleo dorsale del nervo vago nell' attivita riflessa del reticolo. Archo. Sci.Biol., 45, 345-364.

BoRrofF A., I967. Configuration of intestinal slow waves obtained by monopolar recording techniques. Amer. J. Physiol., 213, I 57-I62.

Bost. J., 1958. Fistule permanente du reticulum chez les Petits Ruminants. (Technique nouvelle par voie thoracique). J. Physiol. (Fr.), 50, I70-1 74 .

BozLer E., 1947. The response of smooth muscle to stretch. Amer. J. Physiol., 149, 299-301.

Bulbring E., I955. Correlation between membrane potential, spike discharge and tension in smooth muscle. J. Physiol. (G.B.), 128, 200-221.

Bulbring E., 1957. Changes in configuration of spontaneously discharged spike potential from smooth muscle of the guinea pig's taenia coli. The effect of electrotonic currents and of adrenaline, acetylcholine and histamine. J. Physiol. (G. B.), 135, 412-425. 
Bulbring E., 1962. Electrical activity in intestinal smooth muscle. Physiol. Rev., 42, suppl. 5, 160-1 78.

Burnstock G., Holman M. E., r96r. The transmission of excitation from autonomic nerve to smooth muscle. J. Physiol. (G. B.), 155, II 5-133.

Burnstock G., Holman M. E., Prosser C. L., 1963. Electrophysiology of smooth muscle. Physiol. Rev., 48, 482-521.

Dewey M. M., 1965. The anatomical basis of propagation in smooth muscle. Gastroenterology, 49, 395-402.

DUNCAN D.L., I 953. The effects of vagotomy and splanchnotomy on gastric motility in the sheep. J.Physiol. (G. B.), 119, I 57-169.

DUSSARDIER. M., 1960. Recherches sur le contróle bulbaire de la motricité gastrique chez les Ruminants. Thèse doct. Sci. Paris. I. N. R. A. édit., r96 pp.

FunAKI S., Bohr D. F., I964. Electrical and mechanical activity of isolated vascular smooth muscle of the rat. Nature (Lond.), 208, 192-193.

GARRET J., 1963. Selective blockade of sympathetic or parasympathetic ganglion. Arch. int. Pharmacodyn., 144, 38I-39I.

GiLlesPIE J. S., I962. Electrical and mechanical responses of intestinal smooth muscle cells to stimulation of their extrinsic parasympathetic nerves. J. Physiol. (G. B.), 162, 76-92.

Gonella J., rg64. Étude de l'activité électrique des fibres musculaires longitudinales du duodénum in vivo. Action de la stimulation des nerfs vagues. $C$. R. Soc. Biol., 158, 2409-24I3.

GONELLA J., I965. Variation de l'activité électrique spontanée du duodénum de Lapin avec le lieu de dérivation. C. R. Acad. Sci., 260, 5362-5366.

Holman M. E., I958. Membrane potentials recorded with high resistance micro-electrodes ; and the effects of changes in ionic environment on the electrical and mechanical activity of the smooth muscle of the taenia-coli of the guinea-pig. J. Physiol. (G. B.), 141, 464-488.

IGgo A., LEEK B. F, 1967. An electrophysiological study of single vagal efferent units associated with gastric movements in sheep. J. Physiol. (G. B.), 191, I 77-204.

ITABISAShi T., Matsumoto H., 1966. Electrophysiological studies on the movement of the ruminant stomach. IV. - Relationships between periodic potential fluctuations led from the body surface and from several parts of the stomach walls in goats. Nat. Inst. Arim. Health Quart., 6, 43-55.

Kobayashi M., Prosser C. L., Nagar T., I 667 . Electrical properties of intestinal muscle as measured intracellularly and extracellularly. Amer. J. Physiol., 213, 275-286.

KoIDE F.T., 1966. Determination of the intracellular bridge resistance between smooth muscle cells. Thesis. Ph. D. Univ. Iowa, I64 pp.

LEEK B. F., 1963. Single unit activity in cervical vagal efferent axons associated with reticulo-ruminal movements. J. Physiol. (G. B.), 169, 5P-6P.

MaRShall J. M., 1959. Effects of cstrogen and progesterone on single uterine muscle fibers in the rat. Amer. J. Physiol., 197, 935-942.

Marshall J. M., I962. Regulation of activity in uterine smooth muscle. Physiol. Rev., 42, suppl. 5, 213-227.

Morrison A. R., Habel R. E., I964. A quantitative study of the distribution of vagal nerve endings in the myenteric plexus of the ruminant stomach. J. Comp. Neurol., 122, 297-309.

OHashi H., OHga A., 1967. Transmission of excitation from the parasympathetic nerve to the smooth muscle. Nature (Lond.), 216, $29 \mathrm{I-292}$.

Paton W. D. M., VANE J. R., I963. An analysis of the responses of the isolated stomach to electrical stimulation and to drugs. J. Physiol. (G. B.), 165, I0-46.

Rousseau J.P., I956. Commande motrice vagale de l'estomac et de l'osophage terminal, chez les Ruminants. Róle comparé de la glotte et du sphincter nasopharyngien dans le déterminisme de l'inspiration jorcée de rumination. Thèse Doct. Neurophysiologie, Marseille, 76 pp.

Ruckebusch Y., LAPLACE J. P., 1967. Étude électromyographique de la motricité du complexe gastrique chez le Mouton éveillé. C. R. Soc. Biol. 161, I $5^{81-1588 . ~}$

SPEDEN R. N., I964. Electrical activity of single smooth muscle cells of the mesenteric artery produced by splanchnic nerve stimulation in the guinea-pig. Nature (Lond.), 202, 193-194.

Steedman W. M., rg66. Microelectrode studies on mammalian vascular muscle. J. Physiol. (G. B.), 186, 382-400.

Tomisa T., 1967. Spike propagation in the smooth muscle of the guinea-pig taenia coli. J. Physiol. (G. B.), 181, 517-527.

WOODBURY J. W., BRADY A. J., 1956. Intracellular recording from moving tissues with a flexibly mounted microelectrode. Science, 123, roo-101. 